\title{
BRIEFING NA PRÁTICA SIMULADA: REPRESENTAÇÃO PARA OS ESTUDANTES E DOCENTES
}

Briefing in simulated practice: representation for students and teachers

Briefing in prácticas simuladas: representación para estudiantes y docentes

\author{
Sónia Novais ${ }^{*}$, Maria Alves ${ }^{* *}$, Ana Pinho ${ }^{* *}$, Viviana Baltarejo ${ }^{* *}$
}

\section{RESUMO}

Enquadramento: a prática simulada é uma estratégia pedagógica que estimula a tomada de decisão em casos clínicos. A sua implementação decorre por etapas, sendo o briefing a primeira. Objetivo: compreender a representação do briefing para os estudantes e docentes envolvidos nas aulas de prática simulada do curso de licenciatura em Enfermagem. Metodologia: estudo qualitativo e descritivo orientado pela teoria das representações sociais. Participaram 37 estudantes do curso de Licenciatura em Enfermagem, que se encontravam inscritos em unidades curriculares que recorriam à metodologia de prática simulada e os 7 docentes das mesmas unidades curriculares. A recolha de dados foi realizada através de um questionário que continha um Teste de Associação Livre de Palavras. A analise de dados foi realizada através do software IRAMUTEQ. Resultados: os estudantes representam o briefing valorizando o domínio cognitivo, mas salientando igualmente o domínio afetivo. Os docentes valorizam a preparação dos cenários com o objetivo de alargar o horizonte dos estudantes, procurando aumentar o conhecimento e a experiência através do estabelecimento de objetivos de aprendizagem. Conclusão: considerando os objetivos da prática simulada, o briefing é representado como uma etapa promotora do conhecimento, das aprendizagens e das competências, mas também gerador de emoções.

Palavras-chave: briefing; prática simulada; enfermagem

${ }^{*} \mathrm{PhD}$, Professora Adjunta na Escola Superior de Saúde Norte da Cruz Vermelha Portuguesa/CINTESIS. https://orcid.org/00000002-4328-3755

${ }^{* *}$ Enfermeira, ESSNorteCVP

Como Referenciar:

Novais, S., Alves, M., Pinho, A., \& Baltarejo, V. (2020). Briefing na prática simulada: representação para os estudantes e docentes. Revista de Investigação \& Inovação em Saúde, 3(1), 17-30. doi.10.37914/riis.v3i1.77
Recebido para publicação em: 01/05/2020 Aceite para publicação em: 08/06/2020

\section{ABSTRACT}

Background: simulated practice is a pedagogical strategy that encourages decision-making in clinical cases. Its implementation takes place in stages, the briefing being the first. Objective: to understand the representation of the briefing for students and teachers involved in the simulated practice classes of the Nursing undergraduate course. Methodology: qualitative and descriptive study guided by the theory of social representations. 37 students of the Nursing Degree course participated, who were enrolled in curricular units that used the simulated practice methodology and the 7 teachers of the same curricular units. Data collection was performed using a questionnaire that contained a Free Word Association Test. Data analysis was performed using the IRAMUTEQ software. Results: students represent the briefing valuing the cognitive domain, but also emphasizing the affective domain. Teachers value the preparation of scenarios to broaden the horizon of students, seeking to increase knowledge and experience through the establishment of learning objectives. Conclusion: considering the objectives of the simulated practice, the briefing is represented as a step that promotes knowledge, learning and skills, but also generates emotions.

Keywords: briefing; simulated practice; nursing

\section{RESUMEN}

Marco contextual: la práctica simulada es una estrategia pedagógica que fomenta la toma de decisiones en casos clínicos. Su implementación se lleva a cabo en etapas, siendo el briefing la primera. Objetivo: comprender la representación del briefing para estudiantes y docentes involucrados en las clases de práctica simulada del curso de pregrado en Enfermería. Metodología: Estudio cualitativo y descriptivo. Participaron 37 estudiantes del curso de Grado en Enfermería, que se matricularon en unidades curriculares que utilizaron la metodología de práctica simulada y los 7 maestros de las mismas unidades curriculares. La recopilación de datos se realizó a través de un cuestionario que contenía una prueba de asociación de palabra libre. El análisis de datos se realizó utilizando el software IRAMUTEQ. Resultados: los estudiantes representan la sesión informativa valorando el dominio cognitivo, pero también enfatizando el dominio afectivo. Los maestros valoran la preparación de escenarios para ampliar el horizonte de los estudiantes, buscando aumentar el conocimiento y la experiencia mediante el establecimiento de objetivos de aprendizaje. Conclusión: teniendo en cuenta los objetivos de la práctica simulada, la sesión informativa se representa como un paso que promueve el conocimiento, el aprendizaje y las habilidades, pero también genera emociones.

Palabras clave: briefing; práctica simulada; enfermería 


\section{INTRODUÇÃO}

As Instituições de Ensino Superior da Saúde têm como objetivo principal desenvolver as competências profissionais e pessoais dos estudantes para a futura prática clínica autónoma e responsável. Deste modo, desempenham um papel fundamental no processo de aquisição de competências ao ensinar, acompanhar, estimular e responsabilizar o estudante durante o seu percurso académico.

Uma das estratégias de ensino/aprendizagem que se tem desenvolvido e tem encontrado um grande acolhimento na área da saúde é a simulação, onde a discussão e implementação de cenários clínicos que tentam reproduzir situações reais, permitem que o estudante adquira confiança e mestria para lidar com diferentes situações clínicas problemáticas que futuramente contactará (Hayden, Smiley, Alexander, Kardong-Edgren, \& Jeffries, 2014). A simulação clínica inclui diferentes etapas, o briefing, o cenário e o debriefing (International Nursing Association for \& Clinical Simulation and Learning [INACSL], 2016). O briefing é a primeira etapa e pressupõe o planeamento das dinâmicas de uma prática simulada, sendo definido como a sessão de informações prévia ao cenário de simulação, que inclui as atividades de orientação ao ambiente e revisão dos objetivos e as competências que se pretendem alcançar com o cenário de aprendizagem específico (INACSL, 2016). As atividades realizadas nesta etapa podem ser categorizadas em atividades de orientação ou em atividades de envolvimento e motivação para a aprendizagem que ocorrem antes da implementação prática da simulação (Chamberlain, 2015).
As vantagens da simulação estão descritas em vários estudos realizados neste âmbito (Chamberlain, 2015; Foronda et al., 2016; Mota et al., 2019). Esta estratégia pedagógica permite a discussão e resolução de cenários clínicos, previamente preparados pelos docentes e que promovem o pensamento crítico-reflexivo e a capacidade de tomada de decisão, bem como a gestão do tempo, das emoções e do stress dos estudantes (Jeffries, 2014; Foronda, Liu, \& Bauman, 2016). Outra das grandes vantagens da prática simulada é o facto de os estudantes poderem ter acesso prévio aos cenários e prepararem-se para a discussão e atuação num caso clínico específico, elementos que constituem o briefing (Jeffries, 2014). Considerando que os objetivos da prática simulada devem ser centrados em promover as aprendizagens, o pensamento crítico-reflexivo e a avaliação dos resultados, o briefing assume uma relevância para a sua consecução (Jeffries, 2014).

Foi definido como objetivo para este estudo: compreender a representação do briefing para os estudantes e docentes envolvidos nas aulas de prática simulada do curso de licenciatura em Enfermagem.

\section{ENQUADRAMENTO/FUNDAMENTAÇÃO TEÓRICA}

As Escolas Superiores de Enfermagem devem responder à centralidade do ensino centrado no estudante respeitando a sua diversidade individual e, simultaneamente, devem garantir a qualidade e excelência do ensino, pelo que é importante a adoção de planos flexíveis de aprendizagem e a promoção de competências que estão para além das definidas nos 
currículos formais (European Association for Quality Assurance in Higher Education (ENQA), 2015). Assim, as instituições desempenham um papel fundamental no processo de desenvolvimento de competências ao ensinar, acompanhar, estimular e responsabilizar o estudante durante o seu percurso académico.

A prática simulada é cada vez mais uma estratégia pedagógica utilizada no ensino e aprendizagem em enfermagem, notando-se uma evolução nestas primeiras duas décadas do século XXI (Hayden et al., 2014). São apontadas várias razões para o aumento do recurso à simulação no ensino de enfermagem, incluindo a disponibilidade de simuladores e a suposição de que a aprendizagem num ambiente realista pode ser transferida para a prática clínica sem colocar em risco a vida dos clientes (Hayden et al., 2014).

Com a implementação de programas de simulação nos cursos de enfermagem, os docentes devem garantir que esta seja baseada nas melhores evidências, em particular identificando as fases da simulação que mais influenciam a aprendizagem e o desenvolvimento de competências (Foronda et al. 2013). A INACSL (2016) define o briefing como a sessão de informações que antecede a implementação do cenário de simulação e sugere as atividades para esta etapa, como orientação para o ambiente e a revisão dos objetivos para o cenário específico de aprendizagem. Apesar desta descrição do briefing nos estudos não é apresentado nenhum processo padronizado para o planeamento e implementação desta etapa, diferentemente do debriefing, levando muitos programas de simulação a omiti-lo ou a criarem o seu próprio protocolo (Foronda et al.). Apesar disso, o briefing nas aulas de práticas simuladas, promove o envolvimento e a participação dos estudantes nas atividades de aprendizagem, pelas interações existentes entre estudantes iniciados e os docentes peritos (Sharoff, 2015).

Um componente incluído no briefing é o trabalho em equipa, identificado pela Quality and Safety Education for Nurses (QSEN) como uma competência essencial para a prática clínica de enfermagem. Esta fase motiva os estudantes para o desenvolvimento de competências para gerir o trabalho em equipa e colaborativo (Chamberlain, 2015). O briefing deve incluir a discussão sobre o valor da simulação e dos componentes do debriefing, estabelecendo regras básicas para uma comunicação aberta, fornecendo uma visão geral dos conceitos que o estudante encontrará durante a simulação e fornecendo um ambiente de aprendizagem seguro (Page-Cutrara \& Turk, 2017). Após a etapa de briefing, a simulação com recurso a cenários executados com colegas e supervisionados por docentes qualificados seguidos de técnicas de debriefing apoiam o estudante no desempenho cognitivo, no autoconhecimento e na gestão de emoções potenciando a sua aprendizagem (Mota, et al., 2019; Page-Cutrara \& Turk, 2017; Jeffries, 2014).

A aprendizagem em Enfermagem é fundamentada em três domínios: o cognitivo, que é desenvolvido através da leitura, do estudo e das aulas expositivas e é testado através de questionários escritos ou orais; o psicomotor, que consiste em instruir e treinar procedimentos técnicos em laboratório ou em situações reais; e, finalmente, o afetivo, que decorre das exposições emocionais, culturais e éticas que o estudante encontrará ao longo do curso e na sua 
prática clínica (Jeffries, 2014). Nas aulas de prática laboratorial, o domínio da aprendizagem mais desenvolvido e valorizado é o psicomotor, uma vez que esta tipologia apela a que o estudante aplique o que aprendeu na execução de um procedimento ou na resolução de um caso clínico em equipa. No entanto, sabemos que, para colocar em prática essas aprendizagens e estas se tornarem significativas para o desenvolvimento das competências técnicas e não técnicas, é importante que os estudantes, experimentem e vivenciem uma experiência e emoções próximas das que vivenciariam num cenário real (Jeffries, 2014; Hayden et al., 2014).

Um cenário clínico deve ser previamente planeado pelo docente retratando casos clínicos o mais próximos possível da realidade, de modo a garantir que o estudante coloque em prática o seu processo de tomada de decisão e pensamento critico-reflexivo, as competências de comunicação e de trabalho em equipa ao mesmo tempo que desenvolve procedimentos técnicos, e tendo como finalidade obter sucesso na implementação de cuidados naquela situação específica num futuro profissional (Chamberlain, 2015; Jeffries, 2014). Sendo a tomada de decisão descrita como o equilíbrio entre o pensamento crítico e o julgamento e raciocínio clínico, a cognição situada, a aprendizagem localizada e a flexibilidade cognitiva, é importante que o cenário clínico permita alguma liberdade ao docente e ao estudante para conseguirem discutir e refletir sobre as diferentes abordagens ao caso (Jeffries, 2014).

Embora a INACSL divida a experiência de simulação em três fases: briefing, cenário e debriefing, a primeira fase, nem sempre tem merecido a atenção necessária por parte dos docentes que se encontram envolvidos na simulação, nem tem sido foco de tanta atenção no âmbito da investigação (Page-Cutrara \& Turk, 2017; Meakim et al., 2013).

Os componentes essenciais de briefing são a discussão dos detalhes e das expetativas da simulação, fornecimento de informações básicas necessárias, orientação para 0 ambiente de simulação, esclarecimento sobre a atribuição de funções, discussão sobre os detalhes logísticos e o tempo de preparação do estudante (Lioce et al., 2015). Com esta orientação e envolvimento do estudante ao longo do processo, espera-se aumentar a sua satisfação e participação ao longo da sessão letiva (Chamberlain, 2015). As tarefas realizadas no briefing são atividades preparatórias que o estudante deve concluir antes da atividade de simulação se iniciar, pelo que a clarificação de detalhes e expectativas é uma componente crucial e para além de incluir os componentes essenciais já referido, deve dar espaço para a definição dos termos de confidencialidade, o estabelecimento de um contrato de ficção e a criação de um ambiente de aprendizagem psicologicamente seguro (Rudolph, Raemer, \& Simon, 2014). Ao abordar os detalhes e as expetativas antecipadamente, é menos provável que os estudantes se distraiam ou se sintam nervosos fazendo com que aumente a concentração durante a simulação (Brackney \& Priode, 2014; Rudolph et al., 2014). Neste sentido, abordar a questão da confidencialidade e criar um ambiente psicologicamente seguro durante esta etapa ajudará os estudantes a sentirem-se suficientemente seguros para se envolverem plenamente na simulação e assumir alguns riscos interpessoais durante o 
desenvolvimento do cenário e da fase de debriefing (Rudolph et al., 2014).

A duração da orientação para o ambiente de simulação varia de acordo com a complexidade do cenário clínico, da experiência do docente, do nível do estudante e da familiaridade dos estudantes com a simulação e a configuração simulada (Beischel, 2013). Os estudantes relataram menos ansiedade e melhoraram os resultados da aprendizagem quando os papéis foram estabelecidos e esclarecidos durante o briefing (Beischel, 2013). Quando os docentes aliam o briefing às técnicas de debriefing, o estudante tem a possibilidade de realizar uma retrospeção do que conseguiram implementar e aprender ao longo da simulação facilitando a perceção dos benefícios deste tipo de estratégia de aprendizagem (Page-Cutrara \& Turk, 2017).

Atendendo ao estado da arte sobre o briefing na simulação em enfermagem, a questão de investigação orientadora do estudo é "Qual a representação do briefing na prática simulada dos estudantes de enfermagem e dos docentes?". É objetivo do estudo compreender a representação do briefing para os estudantes e docentes envolvidos nas aulas de prática simulada do curso de licenciatura em Enfermagem. Este estudo é pertinente uma vez que esta etapa é menos explorada no âmbito da investigação, e salienta, o relevo da simulação e do briefing na formação dos futuros enfermeiros.

\section{METODOLOGIA}

Este estudo assume-se num paradigma qualitativo de carácter descritivo e indutivo com orientação da teoria das representações sociais. $\mathrm{O}$ estudo decorreu numa instituição de ensino superior de saúde, após autorização do Conselho de Direção e parecer favorável (Parecer n.o 22) da Comissão de Ética da instituição participante no estudo. Ao longo do estudo foram cumpridos os procedimentos de garantia e salvaguarda dos direitos dos participantes, nomeadamente de voluntariedade, anonimato e confidencialidade.

Através de uma estratégia de amostragem não probabilística de conveniência, foram convidados a participar, através de convite enviado por email, os estudantes do primeiro e segundo ano do curso de licenciatura em enfermagem, que se encontravam inscritos em unidades curriculares que contemplavam na organização da unidade curricular a estratégia pedagógica de prática simulada nas aulas práticas e laboratoriais e os respetivos docentes das mesmas unidades curriculares, pela experiência que estes participantes possuem do fenómeno em estudo. Participaram 37 estudantes, sendo 13 do 1 음 ano e os restantes do 20 ano do curso de licenciatura em enfermagem da instituição em estudo. Participaram ainda, 7 docentes, sendo que os docentes lecionaram aulas de prática simulada simultaneamente nas diferentes unidades curriculares. Todos os docentes participantes possuíam a categoria profissional de professor adjunto.

A recolha de dados foi realizada através de um questionário eletrónico (googledocs) e enviado por email aos participantes pela instituição de Ensino Superior, garantindo a privacidade dos dados no final do primeiro semestre do ano letivo 2018/2019. Na primeira parte, foi realizado um questionário de caraterização do ano que frequentavam ou da 
categoria profissional consoante se tratava do questionário dos estudantes ou o dos docentes. $\mathrm{Na}$ segunda parte, foi realizado um Teste de Associação Livre de Palavras (TALP). Este TALP carateriza-se por ser um teste projetivo e permite a apreensão das representações cognitivas de um grupo social de forma espontânea, revelando conteúdos implícitos ou latentes que podem ser dissimulados nas práticas discursivas (Abric, 2011). No TALP dos estudantes, os estímulos indutores utilizados foram: Quando penso nas aulas de prática simulada lembro-me de; Quando penso nos cenários clínicos preparados para a prática simulada lembro-me de; Quando penso nos objetivos das aulas de prática simulada lembro-me de; Quando penso nos sentimentos/emoções prévias à discussão dos cenários clínicos em aulas de prática simulada lembro-me de; Quando penso nas competências que mobilizo para me preparar para as aulas de prática simulada lembro-me de. No TALP dos docentes, os estímulos indutores utilizados foram: Quando planeio as aulas de simulação lembro-me de; Quando defino os objetivos para os cenários em aulas práticas $e$ laboratoriais lembro-me de; Quando preparo os cenários de prática simulada lembro-me de; Quando penso nas competências que procuro que os estudantes adquiram lembro-me de.

Para análise dos dados recolhidos foi utilizado o software IRAMUTEQ 0.7 alpha 2 (Interface de R pour les Analyses Multidimensionnel- les de Textes et de Questionnaires) que permitiu fazer a análise de dados textuais e de matrizes. Após a preparação do corpus textual, numa base de dados criada no Software Open OfficeCalc versão 4 , foi realizada a análise prototípica. Esta permite que se realize uma análise com recurso a uma matriz que envolve variáveis, categorias e listas de palavras (Camargo \& Justo, 2016). Esta análise, específica do estudo das representações socais, visa identificar a estrutura representacional partindo dos critérios de frequência e ordem de evocação provenientes dos dados dos TALP (Camargo \& Justo, 2016). A análise prototípica permite visualizar, sob forma de um plano fatorial, as palavras organizadas em quatro quadrantes, sendo o primeiro grupo denominado de núcleo central, o 2 e e 3o designados de primeira e segunda periferia, respetivamente, e por fim, um grupo com as palavras menos referidas pelos estudantes e pelos docentes constituindo assim, os elementos de contraste.

Em seguida, foi realizada uma análise de dados com recurso à teoria dos grafos e denominada de análise de similitudes que nos fornece informações sobre a conectividade do discurso, ajudando assim a identificar a estrutura do conteúdo do corpus textual. Também permite identificar as partes e especificidades compartilhadas de acordo com as variáveis descritivas identificadas na análise (Loubère, 2016). Por fim, foi realizada uma análise dos dados segundo o método de classificação hierárquica descendente (CHD) do discurso dos estudantes. Não foi possível a realização desta análise para os docentes pelo baixo número de ocorrências no discurso. Usando matrizes que cruzam formas reduzidas com segmentos de texto, o método de análise da CHD permitiu obter uma classificação definitiva do corpus textual. Os critérios para inclusão dos elementos nas suas classes respetivas é a frequência maior que a média de ocorrências no corpus (Camargo, Bousfield, Giacomozzi, \& Koelzer, 2014). Os resultados são apresentados sob a forma de um dendograma que demostra a relações entre as 
Briefing na prática simulada: representação para os estudantes e docentes

classes, os segmentos de texto e as variáveis dos

participantes fornecendo-lhes um contexto

(Camargo \& Justo, 2016).

\section{RESULTADOS}

Apresenta-se o gráfico de quatro quadrantes (figura

1) do discurso dos estudantes.

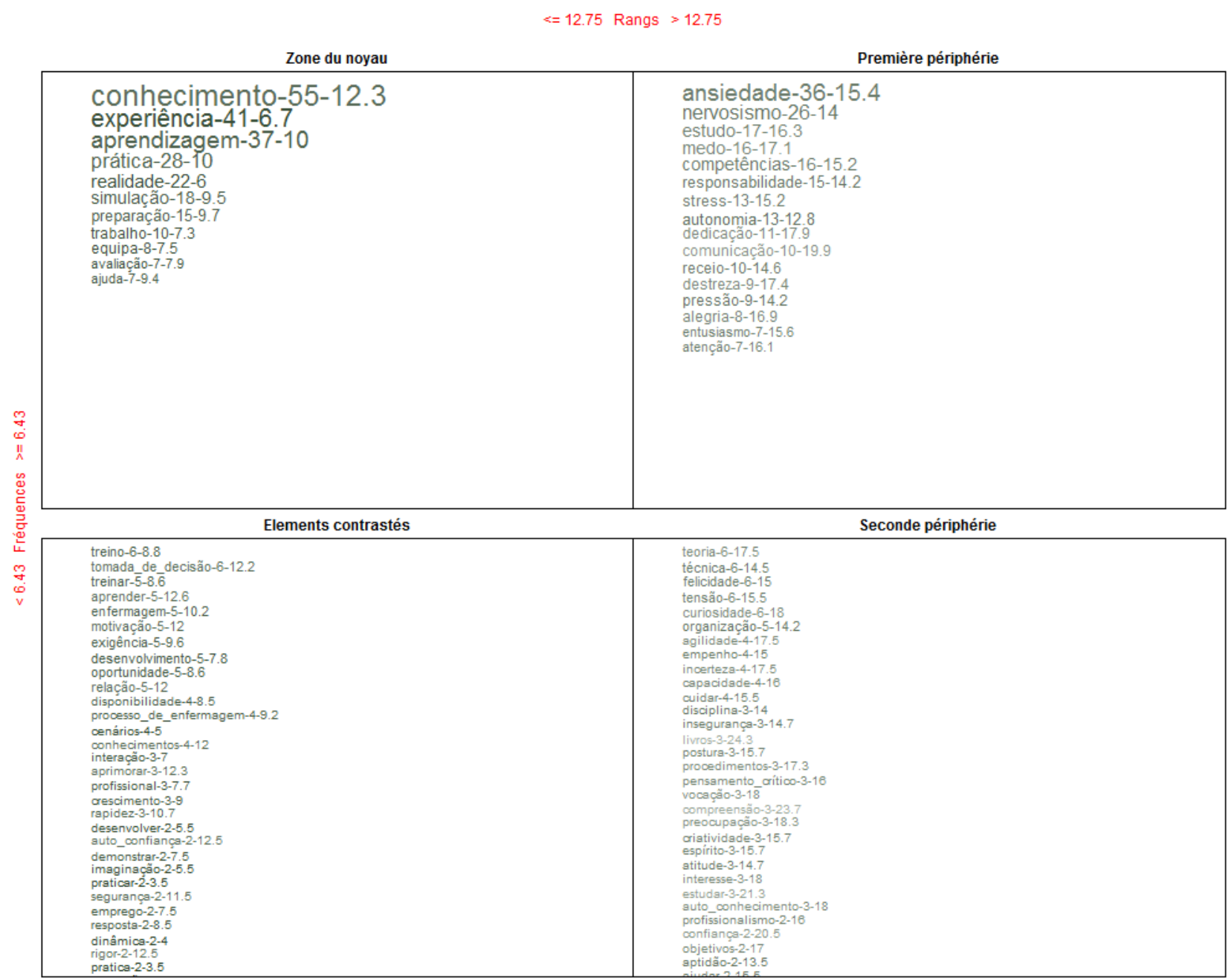

Figura 1

Análise de Prototípica dos estudantes

Destaca-se que, no núcleo central da análise de matriz dos estudantes se encontram mais evocadas as palavras: "conhecimentos", "experiência", "aprendizagem", "prática”, "realidade”, "simulação", "preparação", "trabalho" e "equipa". As palavras mais evocadas na primeira periferia são: "ansiedade", "nervosismo", "estudo", "medo" e "competências". Apresenta-se o gráfico de quatro quadrantes (figura 2) do discurso dos docentes. 


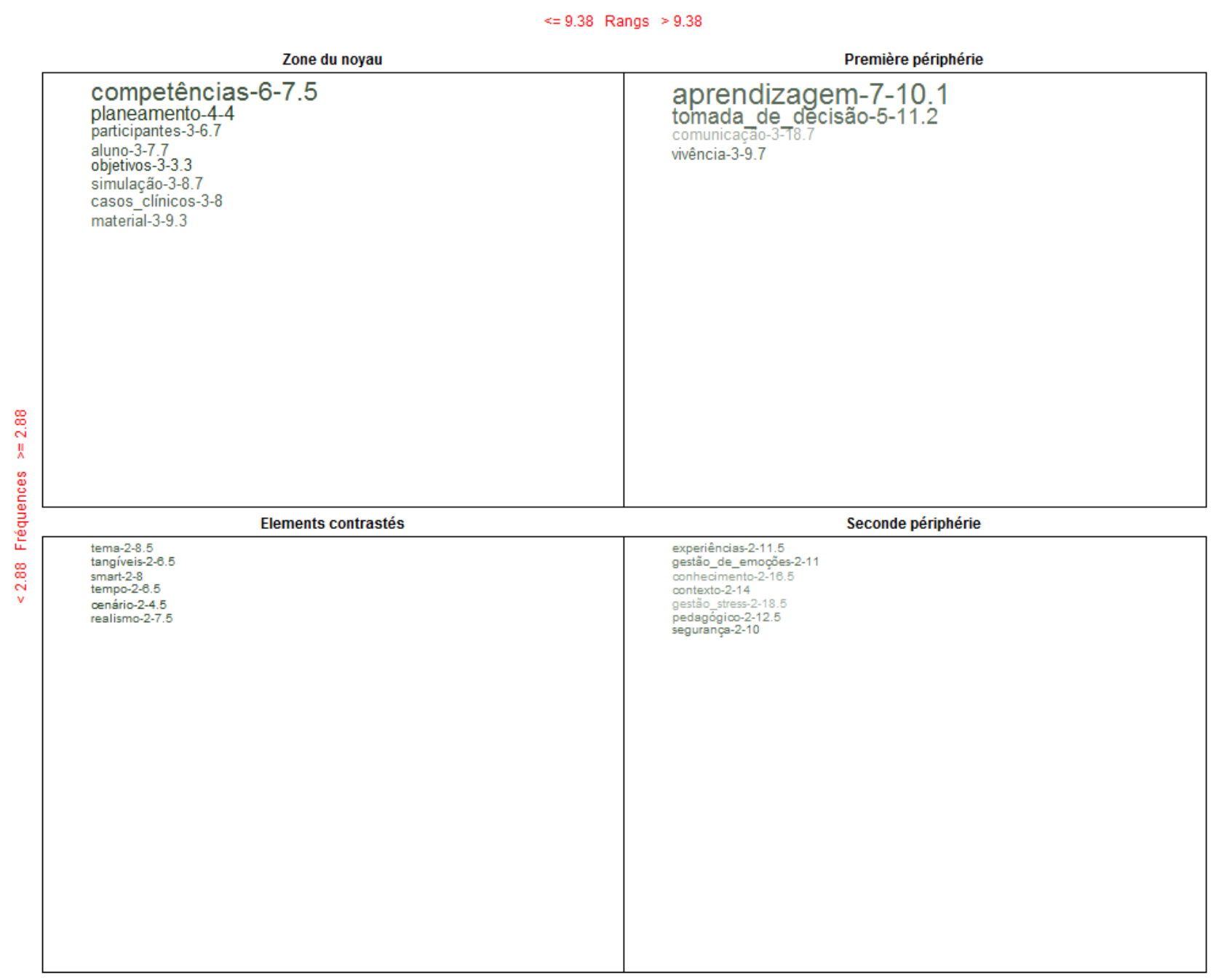

Figura 2

Análise de Prototípica dos docentes

No núcleo central da representação social dos primeira periferia são: "aprendizagem", docentes destacam-se com mais evocadas as "tomada_de_decisão", "comunicação" e "vivência". palavras: "competências", "planeamento", Apresenta-se a árvore das similitudes (figura 3) do "participantes", "alunos" e objetivo. As palavras da discurso dos estudantes. 


\section{Figura 3}

Análise de Similitudes dos estudantes

Percebe-se da sua visualização que o discurso dos "conhecimento", "ansiedade", "experiência", estudantes se organizou em torno de cinco palavras "aprendizagem" e "competência".

com uma relação forte no discurso dos estudantes: Apresenta-se a árvore das similitudes (figura 4) do discurso dos docentes.

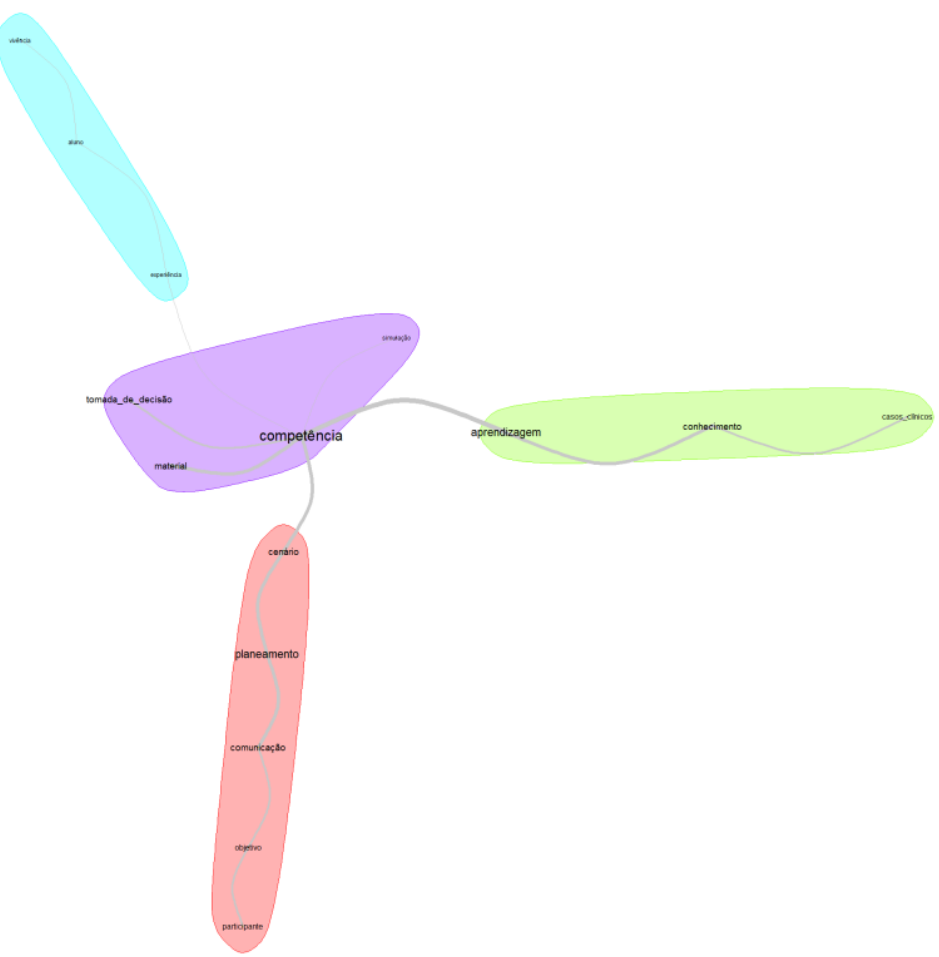

Figura 4

Análise de Similitudes dos docentes 
$\mathrm{Na}$ análise de similitudes relativa aos docentes, percebe-se que a palavra que organiza o discurso com maior relevo é "competência" que apresenta uma relação forte com "aprendizagem", "experiência" e "planeamento".

Os resultados da análise da CHD dos estudantes estão representados pelo dendograma (Figura 5).

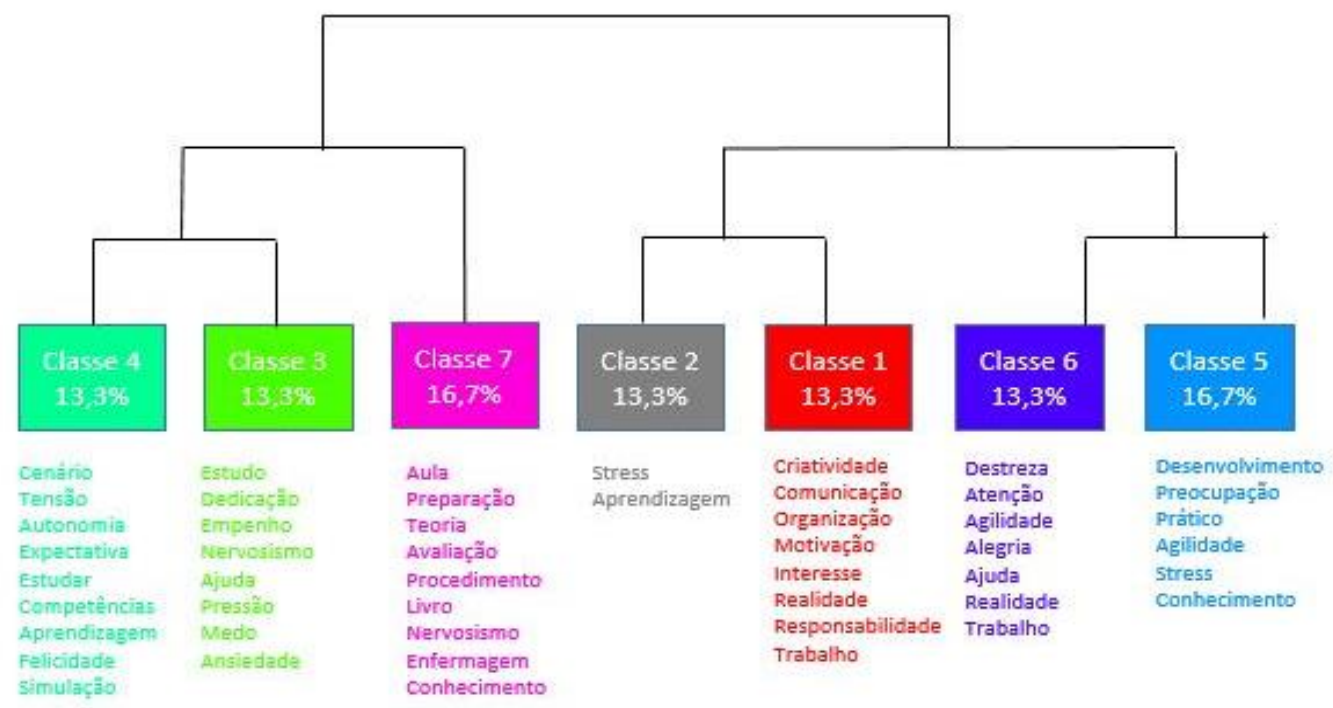

Figura 5

Dendograma da CHD dos estudantes com a relação entre o corpus textual e as classes

No que diz respeito à CHD, verificamos que o corpus textual se dividiu em dois, separando as classes 5, 6, 1 e 2 das classes 7, 3 e 4. No primeiro momento verificamos que o subcorpus textual foi também dividido em dois, separando a classe 5 e 6 da classe 1 e classe 2. Num segundo momento, o subcorpus textual foi também dividido em dois, separando a classe 7 das classes 3 e 4 . Deste modo, consegue-se perceber que o discurso dos estudantes divide-se em duas grandes temáticas, uma relacionada com simulação, ilustrada nomeadamente pelos cenários, o estudo e a própria preparação necessária, outra relacionada com a preocupação dos estudantes pela aprendizagem, quer em termos de destreza e desenvolvimento do seu conhecimento, quer pelo desenvolvimento de competências de comunicação, criatividade e por último com a gestão do stress que motiva a aprendizagem.

A classe 4 foi denominada de briefing é constituída pelas palavras: "cenário", "tensão", “autonomia", "expectativa", "estudar", "competências", "aprendizagem", "felicidade", "simulação". A classe 3 foi designada de domínio afetivo pelas seguintes palavras: "estudo", "dedicação", "empenho", "nervosismo", “ajuda", "pressão", “medo" e "ansiedade". Na classe 7 denominada de domínio cognitivo podemos encontrar palavras como: "aula", "preparação", "teoria”, "avaliação”, "procedimento”, "livro", "nervosismo", "enfermagem" e "conhecimento". A classe 1 foi designada de 
envolvimento na aprendizagem e estão presentes palavras como "criatividade", "comunicação", "organização", "motivação", "interesse", "realidade”, "responsabilidade" e "trabalho". A classe 2 foi designada de mediação dos resultados e apenas apresenta as palavras "stress" e "aprendizagem". A classe 6 designada de domínio psicomotor e apresenta as seguintes palavras: "destreza", "atenção", "agilidade", “alegria", “ajuda", "realidade” e "trabalho". A classe 5 foi designada de resultados e apresenta as palavras "desenvolvimento", "preocupação", "prático", “agilidade" e "conhecimento".

\section{DISCUSSÃO}

Os estudantes, nas diferentes análises textuais ao seu discurso valorizam o "conhecimento" como tendo uma relação estreita com a prática simulada e o desenvolvimento de "competências". Para estes participantes, a "experiência" adquirida por esta estratégia pedagógica é geradora de "aprendizagem". Mas, em contraponto, este "conhecimento" que é exigido é gerador de "ansiedade" pelo sentido de "responsabilidade" percebida.

Por outro lado, os docentes centram-se nas etapas da simulação que visam promover nos estudantes a "aprendizagem", dando enfase à "competência" e ao "planeamento". Na relação estabelecida através da análise de similitudes do discurso destes participantes, nota-se que a "competência" é central nas relações com as restantes comunidades de palavras, nomeadamente com as palavras "aprendizagem", "experiência" e "planeamento". Podemos verificar que os estudantes centram o seu discurso na expectativa dos resultados que advêm da simulação, sejam a vivência de sentimentos e emoções, sejam o desenvolvimento de competências e de aprendizagens.

Verifica-se que os estudantes da instituição em estudo evocam sentimentos de ansiedade que são contrários aos que foram descritos no estudo realizado por Elfrink e colaboradores (2009) onde estes relataram menos ansiedade e melhoraram a aprendizagem quando os papéis foram estabelecidos e esclarecidos durante o briefing.

Olhando para os resultados da CHD percebe-se os estudantes entendem que existe uma relação entre o desenvolvimento de competências e o domínio afetivo na prática simulada, uma vez que o domínio afetivo decorre das exposições emocionais, culturais e éticas que o estudante encontrará na sua prática clínica e pode promover ou inibir o desenvolvimento de competências (Huseb $\emptyset$, Friberg, Søreide, \& Rystedt, 2012). O domínio afetivo é uma dimensão valorizada pelos estudantes na representação que têm do briefing e da prática simulada, mas as palavras que representam esta dimensão aparecem fundamentalmente na análise prototípica na $1 \underline{a}$ periferia. Observando atentamente os resultados desta análise verifica-se que no núcleo central, as palavras que 0 constituem se relacionam principalmente com o domínio cognitivo. A palavra "conhecimento" surge como central também na análise de similitudes dos estudantes e o restante discurso organiza-se em seu torno. Assim, afirma-se que os estudantes valorizam o domínio cognitivo e neste sentido para estes o briefing fornece-lhes informação sobre os objetivos do cenário clínico, os papéis e tarefas do estudante, atribuição de tempo 
para implementar e gerir o cenário, e dá-lhes oportunidade de mobilizarem e aplicarem os conhecimentos aprendidos previamente (PageCutrara \& Turk, 2017).

Através da análise dos resultados dos estudantes e dos docentes contata-se que existem diferenças na representação sobre o briefing dos diferentes participantes relativamente ao seu conceito e à sua importância na prática simulada. Entende-se que, na perspetiva dos estudantes o briefing e a prática simulada, embora promovam o conhecimento e as suas competências também despertam as suas emoções. No entanto, os docentes centram a sua representação na promoção das competências técnicas e científicas que pretendem que os estudantes adquiram e, posteriormente mobilizem nas experiências clínicas. Contudo, também há conformidade de palavras entres os docentes e os estudantes nomeadamente: “experiência” e "competência". Os estudantes também revelam valorizar a importância das experiências clínicas apresentadas pelos docentes que compõem o cenário e a sua discussão. Assim, entende-se que a interação entre o docente e o estudante, imediatamente antes da experiência de simulação, promove a preparação e o envolvimento do estudante no ambiente de aprendizagem (Tyerman, Luctkar-Flude, Graham, Coffey, \& Olsen-Lynch, 2016). Esta interação permite a aplicação das diferentes etapas durante o briefing tornando a aula de prática simulada uma experiência mais ativa, permitindo a revisão dos objetivos de aprendizagem, fornecendo orientação para o material e equipamento e compartilhando outras informações pertinentes relevantes à experiência específica da simulação. A preparação prévia dos estudantes que se obtém pela valorização do briefing melhora o julgamento clínico e o pensamento reflexivo e aprimora os resultados da aprendizagem, portanto, é uma ferramenta eficaz para capacitar os estudantes para serem participantes ativos na sua experiência de aprendizagem (Sharoff, 2015). Embora exista um paradoxo de sentimentos de ansiedade com a simulação, a conscientização e a aprendizagem que se obtém através de técnicas de debriefing colocam a simulação como uma estratégia pedagógica fundamental no âmbito da saúde (Mota et al., 2019; Page-Cutrara \& Turk, 2017).

\section{CONCLUSÃO}

Os objetivos da prática simulada devem ser centrados em promover as aprendizagens, o pensamento crítico e reflexivo e a avaliação posterior dos resultados, sendo que a parte inicial da prática simulada se materializa no briefing. Os estudantes que participaram no estudo representam-no como uma etapa que permite a preparação para aplicação do domínio cognitivo e desenvolvimento de competências, mas com uma relação estreita com o domínio afetivo da aprendizagem, demonstrado pela ansiedade antecipada que provoca. Para os docentes, o briefing relaciona-se com o planeamento das atividades e definição dos objetivos e competências que querem promover nos estudantes. Existe apesar disso, na representação de ambos a valorização da experiência e da competência como conceitos relacionados com o briefing e a prática simulada. Este estudo apresenta como limitação principal o número reduzido de participantes. Pelo facto de ter sido realizada uma análise da representação social de 
um novo conceito que foi introduzido na instituição, deve-se refletir sobre a solidez do núcleo central da representação, pois pode ocorrer que ainda não tenha decorrido tempo suficiente para que a representação social do briefing se tenha objetivado e ancorado nos participantes estudados o que deve ser perspetivado como uma oportunidade de iniciar um trabalho pedagógico consistente com os estudantes e docentes. Consideramos, desta forma, que era importante a continuidade deste estudo num outro ano letivo e a respetiva comparação com estes resultados, de modo a verificar o sentido da representação.

A criação e validação de protocolos de briefing é uma área que deve ser desenvolvida no âmbito da investigação e da pedagogia para que exista a possibilidade de avaliar objetivamente o impacte desta etapa no sucesso da simulação.

\section{REFERÊNCIAS BIBLIOGRÁFICAS}

Abric, J.C. (2011). Méthodologie de recueil des représentations sociales. In J.-C. Abric (Ed.), Pratiques Sociales et Représentations (pp. 73-102). Paris: Presses Universitaires de France.

Beischel, K.P. (2013). Variables Affecting Learning in a Simulation Experience. Western Journal of Nursing Research, 35(2), 226-247. https://doi.org/10.1177/0193945911408444

Brackney, D.E., \& Priode, K.S. (2014). Creating context with prebriefing: A case example using simulation. Journal of Nursing Education and Practice, 5(1). https://doi.org/10.5430/jnep.v5n1p129

Camargo, B.V., Bousfield, A.B.S., Giacomozzi, A.I., \& Koelzer, L.P. (2014). Representações sociais e adesão ao tratamento antirretroviral. Liberabit, 20(2), 17294827. Retrieved from http://revistaliberabit.com/es/revistas/RLE_20_2_re presentacoes-sociais-e-adesao-ao-tratamentoantirretroviral.pdf
Camargo, B.V., \& Justo, A.M. (2016). Tutorial para uso do software IRAMUTEQ (Interface de $R$ pour les Analyses Multidimensionnelles de Textes et de Questionnaires). Retrieved from http://www.iramuteq.org/documentation/fichiers/T utorial IRaMuTeQ em portugues_17.03.2016.pdf

Chamberlain, J. (2015). Prebriefing in Nursing Simulation: A Concept Analysis Using Rodger's Methodology. Clinical Simulation in Nursing, 11(7), 318-322. https://doi.org/10.1016/j.ecns.2015.05.003

Elfrink, V.L., Nininger, J., Rohig, L., \& Lee, J. (2009). The case for group planning in human patient simulation. Nursing Education Perspectives, 30, 83-86.

European Association for Quality Assurance in Higher Education (ENQA). (2015). Standards and Guidelines for Quality Assurance in the European Higher Education Area ESG. Brussels. Retrieved from https://enqa.eu/wpcontent/uploads/2015/11/ESG_2015.pdf

Foronda, C.L., Swoboda, S., Hudson, K., Jones, E., Sullivan, N., Ockimeyf, J., \& Jeffries, P. (2016). Evaluation of vSIM for Nursing ${ }^{\mathrm{TM}}$ : A Trial of Innovation. Clinical Simulation in Nursing, 12(4), 128-131. https://doi.org/dx.doi.org/10.1016/j.ecns.2015.12.00 6

Hayden, J.K., Smiley, R., Alexander, M., KardongEdgren, S., \& Jeffries, P.R. (2014). The NCSBN National Simulation Study: A Longitudinal, Randomized, Controlled Study Replacing Clinical Hours with Simulation in Prelicensure Nursing Education. Journal of Nursing Regulation, 5(2), S3-S40. https://doi.org/10.1016/S2155-8256(15)30062-4

Huseb $\varnothing$, S.E., Friberg, F., Søreide, E., \& Rystedt, H. (2012). Instructional Problems in Briefings: How to Prepare Nursing Students for Simulation-Based Cardiopulmonary Resuscitation Training. Clinical Simulation in Nursing, 8(7), e307-e318. https://doi.org/10.1016/j.ecns.2010.12.002

International Nursing Association for, \& Clinical Simulation and Learning [INACSL]. (2016). INACSL Standards of Best Practice: SimulationSM Simulation Glossary. Clinical Simulation in Nursing, 12, S39-S47. https://doi.org/10.1016/j.ecns.2016.09.012

Lioce, L., Meakim, C.H., Fey, M.K., Chmil, J.V., Mariani, B., \& Alinier, G. (2015). Standards of Best Practice: Simulation Standard IX: Simulation Design. Clinical Simulation in Nursing, 11(6), 309-315. https://doi.org/10.1016/j.ecns.2015.03.005 
Loubère, L. (2016). L'analyse de similitude pour modéliser les CHD. In Actes Des 13eme Journées Internationales d'Analyse Statistique Des Données Textuelles. JADT 2016.

Meakim, C., Boese, T., Decker, S., Franklin, A.E., Gloe, D., Lioce, L., ... Borum, J. C. (2013). Standards of Best Practice: Simulation Standard I: Terminology. Clinical Simulation in Nursing, 9(6), S3-S11. https://doi.org/10.1016/j.ecns.2013.04.001

Mota, L., Maia, C., Soares, F., Marreiros, T., Silva, A.R., \& Freitas, R. (2019). Perspetiva dos estudantes e docentes acerca do debriefing na prática simulada. Revista de Investigação \& Inovação Em Saúde, 2(1), 41-50. https://doi.org/10.37914/riis.v2i1.46

Page-Cutrara, K., \& Turk, M. (2017). Impact of prebriefing on competency performance, clinical judgment and experience in simulation: An experimental study. Nurse Education Today, 48, 7883. https://doi.org/10.1016/j.nedt.2016.09.012

Rudolph, J.W., Raemer, D.B., \& Simon, R. (2014). Establishing a Safe Container for Learning in Simulation. Simulation in Healthcare: Journal of the Society for Simulation in Healthcare, 9(6), 339-349. https://doi.org/10.1097/SIH.0000000000000047

Sharoff, L. (2015). Simulation: Pre-briefing Preparation, Clinical Judgment and Reflection. What is the Connection? Journal of Contemporary Medicine, 5(2). https://doi.org/10.16899/ctd.49922

Tyerman, J., Luctkar-Flude, M., Graham, L., Coffey, S., \& Olsen-Lynch, E. (2016). Pre-simulation preparation and briefing practices for healthcare professionals and students. JBI Database of Systematic Reviews and Implementation Reports, 14(8), 80-89. https://doi.org/10.11124/JBISRIR-2016-003055 